\title{
Alterstice
}

Revue internationale de la recherche interculturelle

International Journal of Intercultural Research

Revista International de la Investigacion Intercultural

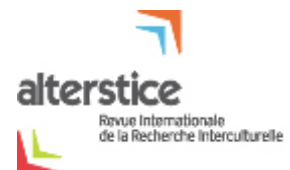

\section{Le plurilinguisme, un nouveau champ ou une nouvelle idéologie ? Ou quand les discours politiquement corrects prônent la diversité}

\section{Aline Gohard-Radenkovic}

Volume 2, numéro 1, 2012

Stratégies de (re)médiation en situation plurilingue. Études de cas en contextes de recherche et d'éducation

URI : https://id.erudit.org/iderudit/1077556ar

DOI : https://doi.org/10.7202/1077556ar

Aller au sommaire du numéro

Éditeur(s)

Alterstice

ISSN

1923-919X (numérique)

Découvrir la revue

Citer cet article

Gohard-Radenkovic, A. (2012). Le plurilinguisme, un nouveau champ ou une nouvelle idéologie ? Ou quand les discours politiquement corrects prônent la diversité. Alterstice, 2(1), 89-102. https://doi.org/10.7202/1077556ar

\section{Résumé de l'article}

Dans cet article, nous identifions les conséquences des discours politiquement corrects qui s'attachent à " reconnaître et promouvoir la diversité » sur le champ scientifique des langues et des cultures étrangères au sein de l'Union européenne, puis plus spécifiquement en Suisse, pays officiellement plurilingue. Nous nous posons la question : le plurilinguisme traduit-il un nouveau champ scientifique ou une nouvelle idéologie, ou les deux à la fois ? 
CONTREPOINT

\section{Le plurilinguisme, un nouveau champ ou une nouvelle idéologie? Ou quand les discours politiquement corrects prônent la diversité}

Aline Gohard-Radenkovic ${ }^{1}$

\section{Résumé}

Dans cet article, nous identifions les conséquences des discours politiquement corrects qui s'attachent à " reconnaître et promouvoir la diversité » sur le champ scientifique des langues et des cultures étrangères au sein de I'Union européenne, puis plus spécifiquement en Suisse, pays officiellement plurilingue. Nous nous posons la question : le plurilinguisme traduit-il un nouveau champ scientifique ou une nouvelle idéologie, ou les deux à la fois?

\section{Rattachement de l'auteure \\ ${ }^{1}$ Université de Fribourg, Fribourg, Suisse}

\section{Correspondance}

aline.gohard@unifr.ch

\section{Mots clés}

plurilinguisme; discours sur la diversité; idéologies de la pluralité; discours "linguistico-politiquement corrects »; processus de légitimation

\section{Pour citer cet article :}

Gohard-Radenkovic, A. (2012). Le plurilinguisme, un nouveau champ ou une nouvelle idéologie? Ou quand les discours politiquement corrects prônent la diversité. Alterstice, 2(1), 89-102. 


\section{Introduction}

À la clôture du Congrès de l'ARIC à Florianopolis ${ }^{1}$, j'ai eu l'occasion de présenter les principes et les démarches qui ont fondé l'élaboration collaborative du Précis du plurilinguisme et du pluriculturalisme (Zarate, Lévy et Kramsch, 2008, qui a été traduit en anglais en 2011). J'avais souligné les " conclusions prudentes » de cette expérience de mises en commun et de confrontations conceptuelles, qui avait réuni 90 chercheurs, spécialistes du domaine des langues et cultures, et plus de 30 institutions différentes du monde entier, dont les rencontres scientifiques avaient été rythmées par des séminaires et colloques de 2003 à 2007. Il s'agissait d'une exploration qui se concevait sur la durée, toujours en chantier, inachevée, qui a participé (et participe encore) à l'émergence d'un champ complexe, en devenir, en contradiction, en tension, en interrogation : le plurilinguisme. Démarche donc de facto plurilingue, pluriculturelle, plurinationale, pluridisciplinaire, etc. mais qui ne prétend pas revendiquer, à tout prix, «le respect et la promotion de la diversité ». Et d'ailleurs, pour se démarquer des termes à la mode - et des idéologies qu'ils véhiculent -, dès l'introduction c'est le terme " pluralité » qui sera adopté et non celui de " diversité », utilisé $a d$ nauseam...

Par ailleurs les trois éditrices du Précis rappellent aussi que :

Le temps passé aux débats a permis de mettre au jour des postures de recherche qui sont souvent enfouies dans les échanges scientifiquement formatés. Dans les débats, les règles tacites de la circulation internationale des idées où « les textes circulent sans leur contexte" (Bourdieu, 2002) sont apparues clairement quand le concept est extrait du contexte social et historique immédiat dont il est le produit, quand il est soumis dans son contexte d'accueil à des distorsions où il est difficile de retrouver le débat initial. (Zarate, Lévy et Kramsch, 2008, p. 21-22)

Dans cet article nous essaierons d'identifier les conséquences des discours politiquement corrects qui s'attachent à " reconnaître et promouvoir la diversité » sur le champ scientifique des langues et des cultures étrangères au sein de l'Union européenne, puis plus spécifiquement en Suisse, pays officiellement plurilingue. Enfin, nous situerons la didactique des langues et des cultures dans ces luttes de positions, passées et présentes, et nous poserons la question : le plurilinguisme traduit-il un nouveau champ scientifique ou une nouvelle idéologie?

\section{Le champ ou le « chant » du plurilinguisme dans les discours européens?}

Si les politiques européennes sont soucieuses de vouloir développer des compétences plurilingues dans l'expérience de mobilité - et dans la foulée des compétences pluriculturelles (parfois interculturelles : on surfe sur les termes) - et déclinent toutes les procédures et tous les procédés possibles pour atteindre le "nirvana didactique " dans le domaine, c'est parce qu'elles sont étroitement liées à un postulat d'ordre éthique, en fait idéologique, celui du "respect de la diversité ", impliquant un droit à la reconnaissance de "sa/ses » langue(s) et de « sa/ses » culture(s).

II suffit de regarder le haut de la page couverture du Guide pour l'élaboration des politiques linguistiques éducatives en Europe, publié par le Conseil de l'Europe (2008) - on peut y lire " De la diversité linguistique à l'éducation plurilingue »- pour en comprendre l'impact. Cette idéologie est donc affichée noir sur blanc: elle sous-tend tous les euro-discours de ces dix dernières années.

Mais, en premier lieu, nous nous poserons la question suivante : quelles sont les conséquences observables « du respect et de la reconnaissance de la diversité linguistique » sur le fonctionnement des institutions de l'Union européenne?

Le "respect de la diversité des langues » : conséquences sur les (dys)fonctionnements de l'Union européenne

Nous rejoignons Calvet (2002) qui démonte la grande machine européenne à fabriquer du "politicolinguistiquement correct » (PLC) à travers les discours et divers dispositifs de la Commission européenne.

${ }^{1}$ Congrès de l'ARIC à Florianopolis, Brésil, juin-juillet 2009. 
Rappelons-en avec cet auteur (Calvet, 2002, p. 43-45) les principaux jalons :

- le régime linguistique de l'Union européenne (UE) est fixé par le traité de Rome en 1957 : langues officielles des pays membres serait ipso facto langues officielles de la communauté, soit 6 : Allemagne, Belgique, France, Italie, Luxembourg, Pays-Bas (sauf le luxembourgeois);

- d'autres pays vont adhérer à l'UE : Danemark, Grande-Bretagne et Irlande en 1973; Grèce en 1981; Espagne et Portugal en 1986 : 15 États membres avec 11 langues officielles (sauf le gaëlique);

- le nombre de combinaisons de traductions possibles pour 11 langues s'élève donc à 110 combinaisons! Le Service d'interprétariat compte près de 500 interprètes stables sans compter les 200 à 300 en free lance. II assure l'interprétation de 40 à 50 réunions par jour ce qui fait au total 125000 journées-interprètes par an... ce qui correspond à $60 \%$ du budget annuel de fonctionnement de l'UE...

Et de nos jours, que se passe-t-il avec les 27 pays membres de l'UE? Quelles sont les difficultés rencontrées dans ce processus de babélisation?

On se heurte souvent à ces difficultés : comment trouver des interprètes pour certaines langues (ex. grec-finnois)? On a recours à des langues-pivots: grec-français-français-anglais-anglais-finnois avec bien sûr une grande déperdition sémantique à chaque étape de la traduction. Parallèlement le nombre des textes écrits augmente de manière régulière car les défenseurs des «langues menacées ", s'appuyant sur le principe que " toutes les langues sont égales » et donc que " toutes les langues doivent être écrites » (Calvet 2002, p. 107), accentuent la lourdeur du système et accélèrent même la paralysie de l'institution. Nous sommes d'accord avec Calvet (2002) quand il conclut que cette démultiplication des langues, accompagnée d'une politique de régionalisation - Europe des régions au-delà des États-nations - sous couvert de "reconnaissance de la diversité linguistique ", tient de la déraison voire de la folie...

En d'autres termes, la question linguistique à Bruxelles est devenue ingérable, une véritable " patate chaude » dira Calvet (2002, p. 44), qui conclut que « ce respect de la diversité est un jeu à somme nulle dans lequel les gains et les pertes s'annulent» (p.46). Pourquoi? Parce que la promotion d'une langue se fait toujours au détriment des autres. Pire encore, on est dans le processus de fabrication de langues légitimes - et d'autres non légitimes - et des "cultures » qui leur sont liées, engendrant des processus politiques de (re)légitimation-déligitimation - et donc d'exclusion-inclusion - de plus en plus incontrôlables.

\section{Le "plurilinguisme à tout prix " ou l'art de fabriquer du nouveau à partir de l'ancien}

Depuis une dizaine d'années, qu'observons-nous dans le champ des langues et des cultures? Un « plurilinguisme en construction ». Loin d'user d'une prudence tant conceptuelle que méthodologique qui respecterait la déontologie scientifique prônée dans le Précis (voir ci-dessus), un grand nombre de domaines, de disciplines, d’instituts, de départements, de cursus entiers ou de spécialisations se déclarent et s'auto-déclarent " pluri-quelque chose ", sous des dénominations telles que "plurilinguisme ", "sciences du plurilinguisme ", " didactique du plurilinguisme », "éducation plurilingue», "Multilingualism and Multiculturalism», recourant à l'anglais pour faire plus " authentique » ou plus " actuel ", annonçant du nouveau tout en maintenant d'anciennes situations (par exemple, côtoiements ou cloisonnements disciplinaires) et véhiculant des conceptions scientifiques qui relèvent davantage de convictions et de modes que d'analyses distanciées.

Depuis la parution du Guide pour l'élaboration des politiques linguistiques éducatives en Europe (Conseil de l'Europe, 2008), nous assistons à une prolifération de ces termes, que théoriciens et praticiens utilisent et didactisent sans bien toujours savoir ce qu'ils recouvrent. Cela nous rappelle étrangement le sort de "l'interculturel », cette « insoutenable innocence de l'interculturel » comme l'écrit Christian Giordano (2008), qui a fait les choux gras d'un grand nombre d'institutions et de formations, tant publiques que privées, car si l'interculturel est un terme accrocheur, le plurilinguisme est lui aussi très vendeur et peut rapporter beaucoup d'argent, attirer des subventions et susciter des bénédictions... 
Il ne s'agit pas de remettre en question le "plurilinguisme » et le "pluriculturalisme » en tant que tels: il est indispensable d'en analyser les configurations et les situations à différents niveaux (politique, économique, social, institutionnel, groupal et individuel). II s'agit plutôt ici d'interroger leurs usages, leurs conceptions, leurs interprétations (comme l'ont fait systématiquement les auteurs du Précis), sans oublier leurs excès, leurs détournements, leurs positions implicites et leurs impostures: bref, analyser ce qu'on appelle la nouvelle doxa (discours dominant) en vigueur et identifier les acteurs qui la relaient (ou ont intérêt à la relayer) à différents niveaux de l'institution et de la société, ceux que Pierre Bourdieu désigne comme les clercs (1980).

\section{Les chantres du plurilinguisme : idéologie contre idéologie}

Bruno Maurer (2011) dresse, quant à lui, un bilan critique de l'enseignement des langues et cultures sous I'influence des recommandations du CECR (1998), qui encourage le développement d'une éducation plurilingue et de surcroît interculturelle, garante de l'«intercompréhension linguistique " et de la communication tant " dialogique qu'harmonieuse » entre les peuples.

L'auteur rappelle qu'une idéologie monolingue a longtemps sévi en Europe, dans des États-nations qui, ne l'oublions pas, se sont construits les uns contre les autres avec, pour arme majeure de cohésion nationale entre leurs citoyens, une seule et unique langue d'identification. Le bi/plurilinguisme - vécu le plus souvent sur le mode individuel - est alors considéré comme bizarre, anormal, voire " nocif » (Tabouret-Keller, 2011), et parfois même ressenti comme une menace. On voit ici la part de l'idéologie dans la lecture des réalités sociales et individuelles. Toutefois, Maurer constate que, depuis plus d'une décennie, les politiques de I'UE, relayées par le Conseil de l'Europe, promeuvent une idéologie plurilingue qui est en train de remplacer l'idéologie monolingue, avec les mêmes tours de force de lecture qu'elle offre du réel (p. 66).

Pour montrer les effets pervers de ces idéologies «naïves " qui circulent de manière le plus souvent inconsciente, même chez des chercheurs avertis, il cite Peter Lenz et Raphaël Berthele (2010, soulignement de Maurer) :

Comme nous l'avons déjà mentionné dans le premier chapitre, l'éducation plurilingue vise principalement à lever les barrières linguistiques artificielles entre les langues - barrières qui restent profondément ancrées dans l'esprit de nombreux locuteurs, enseignants, évaluateurs en langues - et à promouvoir l'usage intégral d'un répertoire que possèdent de fait la plupart des gens.

Maurer commente ce passage ainsi :

En quoi cette phrase est-elle une interprétation idéologique du réel? Elle minimise volontairement les différences entre les langues, pourtant fruits de siècles d'évolution divergente ou résultat d'appartenances à des familles linguistiques différentes, en posant que celles-ci seraient des " barrières artificielles », donc créées de toutes pièces. Loin de nous l'idée qu'il ne faille pas encourager les gens à rechercher les points de convergence, les ressemblances. À les décomplexer de leurs apprentissages, mais de là à prétendre qu'il existerait des barrières artificielles qu'il s'agirait juste de lever [...]. (p. 66-67)

Cette lecture du réel repose donc sur plusieurs affirmations qui sont autant de partis pris idéologiques, la plus notable étant «l'évidence du plurilinguisme ». De notre côté, nous avons pu identifier dans les (euro)discours ces " processus de naturalisation " qui affectent non seulement les conceptions de "cultures " et d'« interculturel " (Gohard-Radenkovic, 2006), mais aussi celles du plurilinguisme (Gohard-Radenkovic, 2010), et nous avons montré que les discours, tant savants que profanes, niaient l'impact des rapports de force et conflits sur le statut des langues et sur le rapport entre les langues tant au niveau national qu'international, et tant au niveau individuel qu'institutionnel.

\section{Ces discours " politiquement corrects " sur la diversité : leurs effets sur le champ de la didactique des langues et cultures étrangères}

Depuis la restructuration des cursus selon Bologne au début des années 2000, qui a concerné l'ensemble du système universitaire européen, la didactique des langues et des cultures étrangères - domaine jusqu'alors peu visible, car dépendant d'autres disciplines (littérature, linguistique, sciences de l'éducation ou pédagogie) - a pris progressivement son autonomie et connaît un développement sans précédent, porté par les politiques de UE. 
En quoi ces discours politiquement corrects sur la diversité ont-ils eu un impact sur la reconnaissance ou la nonreconnaissance de ce nouveau champ et comment s'articulent-ils avec le plurilinguisme?

\section{La didactique des langues et cultures étrangères : une légitimité variable mais en pleine expansion}

Nous avons pu constater que le statut de ce domaine est très variable d'un pays à un autre et d'une discipline à une autre: ainsi la didactique des langues étrangères, également dénommée " didactologie des langues et cultures étrangères » en France (Galisson, 2002; Galisson et Porcher, 1985; Porcher, 1987), et la "Fremdsprachendidaktik ", dénommée aussi "Sprachlehrforschung » ou "Fremdsprachunterricht » en Allemagne et en Autriche (Bausch, Christ, Königs et Krumm, 1996; Edmonson et House, 2000), existent depuis le milieu des années 1980 avec leurs filières et diplômes jusqu'au doctorat et à l'habilitation. Peu de temps auparavant, des filières universitaires en didactique du français langue seconde (FLS) se sont constituées au Québec, donnant l'exemple d'une didactique préoccupée par l'intégration par la langue des immigrants. La didactique de l'anglais ESL/ELF/ESOL a acquis depuis longtemps un statut universitaire international du fait de l'attraction de sa langue et de ses modèles économiques et culturels, mais aussi en raison de forts taux d'immigration dans les pays anglophones occidentaux.

En réponse à des groupes ou individus qui sont en situation de mobilité ou de migration pour des raisons de plus en plus diversifiées (économique, politique, climatique, éducative, académique, professionnelle) et qui choisissent de nouvelles destinations, ont émergé en cette dernière décennie de nouvelles didactiques. Pour ne citer que quelques chercheurs, en Italie une didactique de l'italien langue seconde/langue étrangère s'est constituée et propose des filières complètes (avec l'Université de Macerata, pionnière, en $2004^{2}$ ); nous avons constaté le même phénomène pour les mêmes raisons en Espagne, en Grèce (Androulakis, 2003), à l'Institut d'études interculturelles à Timisoara (Roumanie, en 2010) ( $^{3}$ et, au-delà de l'Europe, dans les pays d'Asie centrale (universités du Kazakhstan, en $2010^{4}$ ), l'Université Waseda à Tokyo ${ }^{5}$, pionnière quand elle a ouvert en 2001 le premier département de didactique du japonais langue étrangère.

Les didactiques premières ne se sont pas développées uniquement dans leur pays de naissance, mais se sont " exportées » ou plutôt ont été " aménagées » par un certain nombre de pays d’Europe centrale et orientale et plus largement dans le monde. Ces didactiques forment un champ constitué, avec leurs ancrages disciplinaires, leurs courants conceptuels et méthodologiques, etc., propres à l'histoire et aux enjeux du contexte où elles ont été produites et interprétées.

\section{La didactique des langues étrangères dans un pays plurilingue : une légitimité récente, variable et instable}

Qu'en est-il de la Suisse? Cet exemple illustre les conséquences des discours politiquement corrects sur un pays où nous avons un plurilinguisme constitutionnel, datant de 1848. Que se passe-t-il depuis la restructuration des cursus sur le modèle de Bologne, l'adhésion inconditionnelle au CECR par les instances éducatives et l'adoption des « approches plurielles » en didactique des langues?

Bref retour sur le passé. Les didactiques des langues nationales qui sont enseignées au niveau du primaire et de la première année de secondaire existent depuis les années 1980 : ce sont celles du français, de l'allemand et de l'italien (ce n'est pas l'expression "langue étrangère » qui est utilisée, mais " langue seconde »), plus récemment

\footnotetext{
${ }^{2}$ Danièle Lévy et son équipe ont conçu et mis en place le premier Master en didactique de l'ILS en réponse aux besoins de publics migrants (élèves et adultes) qui n'ont cessé de se diversifier depuis les années 1990.

${ }^{3}$ Calin Rus, directeur de cet Institut à l'Université de l'Ouest de Timisoara, a dirigé de 2009 à 2010 une équipe chargée, par le ministère de l'Éducation du Banat, de la conception et de la mise en place pour les adultes migrants d'un programme de formation en roumain langue seconde.

${ }^{4}$ L'implantation, dans les universités du Kazakhstan, de nouvelles didactiques des langues, dont était responsable la professeure Kuliash Duisekova à I'URILM (Almaty), mandatée par le ministère de l'Éducation, a accompagné une nouvelle politique des langues et la restructuration de cursus selon le modèle de Bologne.

${ }^{5}$ Hideo Hosokawa, nippologue et directeur du Centre de langues, a été l'initiateur avec son équipe de cette didactique qui a eu comme objectif de répondre aux besoins des étudiants étrangers, dont le nombre croît depuis deux décennies.
} 
celle de l'anglais. Ces didactiques étaient/sont enchâssées dans la didactique ou la pédagogie générale, qui est tournée vers la formation pratique, et donc n'ont pas été reconnues comme formant un domaine en soi disposant d’un appareil théorique spécifique.

Dans certaines universités francophones (Genève, Lausanne, Neuchâtel), il existait bien une formation en didactique du FLE (français langue étrangère), mais celle-ci a longtemps été traitée en " annexe » et son diplôme n'était pas reconnu par les instances académiques. C'était pire pour le DaF (Deutsch als Fremdsprache [allemand langue étrangère]), dont le concept même était inconnu, car le champ de l'allemand (comme langue maternelle) était/est occupé par les philologues, qui ont pignon sur rue. Ces didactiques en émergence dans le cadre de la politique plurilingue volontariste de la Suisse sont devenues un peu plus «légitimes » depuis la restructuration des cursus selon Bologne, mais, dans la plupart des universités, elles sont encore dominées par les domaines traditionnels (linguistique et littérature).

Les didactiques du FLE et du DaF se sont lentement constituées depuis la fin des années 1990 à l'Université bilingue de Fribourg avec des théories "importées" par des didacticiens de France et d'Allemagne. Elles figurent officiellement depuis 2003 dans les cursus Bachelor et depuis 2006 dans les cursus Master. Plus récemment s'est créé un Domaine du Plurilinguisme ou Mehrsprachigkeitsbereich, dominé par la linguistique (appliquée), et qui cherche ses marques dans la formulation d'une "didactique plurielle ». Si l'Université de Fribourg a été pionnière en promouvant ces didactiques dans le cadre de nouveaux cursus, en revanche la dénomination " didactique " a été prise en otage par l'Institut chargé de la formation pratique des futurs enseignants ${ }^{6}$, toutes disciplines confondues. De quoi en perdre sa didactique...

On peut s'étonner qu'une didactique des langues secondes et étrangères se soit constituée si tardivement dans un pays officiellement plurilingue et dont la préoccupation majeure est la gestion des langues et leur transmission, garantes du projet de cohésion politique et sociale de l'État fédéral (Widmer, Coray, Acklin Muji et Godel, 2004). Nous avons déjà tenté d'expliquer les raisons de ces différences de légitimité (Gohard-Radenkovic, 2005 et 2007 ). Nous voyions alors deux explications à cette difficulté de reconnaissance :

- elle était liée à des représentations héritées des disciplines les unes dites "d'enseignement » et les autres dites "d'intervention", entraînant une conception dichotomique et hiérarchisée de domaines perçus " pratiques » pouvant entraîner une stigmatisation académique (Galisson, 2002);

- et comme cela a été déjà évoqué, elles étaient inféodées à d’autres disciplines (linguistique, littérature), celles de la langue maternelle, perçues comme « nobles " parce que possédant une tradition théorique bien ancrée.

Nous percevons aujourd'hui une troisième explication : elle est liée au fait que ces didactiques nées dans d’autres pays d’Europe ont bénéficié de politiques étatiques et de réseaux de diffusion mondiaux, s'inscrivant dans une mouvance post-coloniale (France, Belgique) ou dans celle de la reconquête d'anciennes influences (Allemagne, Autriche); ces didactiques se sont développées hors des institutions éducatives nationales (non captives), avec des enjeux spécifiques auprès de divers publics. Au contraire, l'apprentissage obligatoire des langues nationales en Suisse dès le primaire a été conçu comme une discipline captive du système éducatif, enseignée à des fins institutionnelles et ne considérant pas les langues comme des outils de communication et d'acquisition de nouveaux savoirs et savoir-faire.

Néanmoins, cette acceptation de nouvelles didactiques symbolise un premier pas vers la reconnaissance de domaines plus ou moins autonomes, avec des préoccupations tant "métadidactiques » que "pragmatiques », destinés à la formation de futurs enseignants bi-plurilingues qui devront en principe assurer la transmission de la langue « partenaire » dans le cadre du plurilinguisme.

\footnotetext{
${ }^{6}$ Redénommé depuis 2008 «Centre d’Enseignement et de Recherche pour la Formation des maîtres » (CERF).
} 


\section{Ces discours PC sur la diversité : des références scientifiques en tension dans le champ de la didactique des langues et cultures étrangères}

\section{Les disciplines de référence pour les différentes didactiques}

Nous l'avons vu, on ne peut pas vraiment pas parler en Suisse de traditions scientifiques en didactique des langues, puisque le champ théorique s'est récemment développé. Toutefois les chercheurs du domaine des langues de I'Université de Fribourg avaient pour mission de fonder une plate-forme conceptuelle commune en vue de la création du domaine du plurilinguisme.

Dans cette optique, une enquête a été menée en 2007 par un collègue angliciste et anglophone, Anthony Clark (2008), sur les références théoriques et méthodologiques que les enseignants de l'ex-CERLE ${ }^{7}$ véhiculaient dans leurs enseignements et leurs recherches.

Notre collègue nous a posé un certain nombre de questions sur des concepts et des pratiques (par questionnaires en anglais et entretiens dans notre langue maternelle), essentiellement issus du TESOL, qu'il jugeait partagés par tous les didacticiens du Centre. Ses conclusions ont été les suivantes :

It is clear that, even between speakers of one language, sometimes technical terms are not shared, for example there may be differing understandings of bilingualism or of multilingualism. In addition some of these definitions are more stable, others still in flux. Nevertheless, it was a firm impression after these interviews that English and German speakers had more common technical vocabulary than the French. Correspondingly, a number of the French-speaking teachers spoke more briefly in response to some questions than did the teachers of German and English [...]

Furthermore, while the data provides good evidence for consensus and shared understandings within language sections, there is much less for such shared understandings across the languages. Although I focussed mainly on possible confusion over terminology, one bilingual teacher (French/German) downplayed such differences and stressed that the main differences were in "ideology".

Ces commentaires, que je qualifierai de "naïfs », révèlent toutefois :

- que les références et conceptions sont «pensées » à partir d'une didactique évidente, celle du TESOL, parce que portée par une langue dominante, l'anglais, ce qui était vrai pour les didacticiens du DaF, tandis que ceux du FLE étaient perçus comme des résistants, voire des rebelles, en tout cas des marginaux;

- que les didacticiens du discours dominant ignorent (ou nient?) jusqu'à l'existence possible d'autres héritages, d'autres ancrages, d'autres conceptions, d'autres autonomies dans le domaine des langues;

- que les didacticiens d'une même langue ont des références conceptuelles communes, une "culture didactique " qui n'offre pas d'homogénéité en soi mais qui présente toutefois un socle de conceptions partagées.

\section{Les ancrages disciplinaires prédominants selon les didactiques}

Derrière ces conceptions prédominantes de la didactique, on trouve des ancrages disciplinaires différents, et donc des positionnements théoriques prédominants différents, ce que nous illustrons dans les figures 1 et 2 ci-dessous.

\footnotetext{
${ }^{7}$ Centre d'enseignement et de recherche en langues étrangères de l'Université de Fribourg qui réunissait alors les trois langues nationales + l'anglais ; ce centre a été scindé en 2006 en deux : avec un centre de langues d'un côté et un domaine académique du plurilinguisme et de la didactique des langues étrangères de l'autre.
} 
Figure 1 : Références disciplinaires prédominantes en didactique du DaF

\begin{tabular}{|c|c|c|}
\hline $\begin{array}{l}\text { EBENE } 3 \\
\text { Unterrichtliche } \\
\text { Praxis }\end{array}$ & $\begin{array}{l}\text { Methodologie } \\
\text { Lehrziele } \\
\text { Lehrinhalte } \\
\text { Lehrmaterialien }\end{array}$ & $\begin{array}{l}\text { Organisation } \\
\text { Planung } \\
\text { Lehrerausbildung } \\
\text { Bildungssystem }\end{array}$ \\
\hline \multirow{3}{*}{$\begin{array}{l}\text { EBENE } 2 \\
\text { Zwischenebene }\end{array}$} & \multicolumn{2}{|c|}{$\begin{array}{c}\text { «EDUCATIONAL LINGUISTICS » } \\
\text { THEORIE und PRAXIS }\end{array}$} \\
\hline & \multicolumn{2}{|c|}{ Lehr-/Lernumgebung } \\
\hline & Lernen & he \\
\hline $\begin{array}{l}\text { EBENE } 1 \\
\text { Grundlagen }\end{array}$ & \multicolumn{2}{|c|}{$\begin{array}{l}\text { Geschichte des Fremdsprachenunterrichts } \\
\text { Linguistik } \\
\text { Soziologie, Anthropologie } \\
\text { Psychologie, Psycholinguistik } \\
\text { Erziehungswissenchaft }\end{array}$} \\
\hline
\end{tabular}

Source : d'après Edmondson et House, 2000, utilisant un modèle de Stern

Figure 2 : Références disciplinaires prédominantes en didactique du FLE

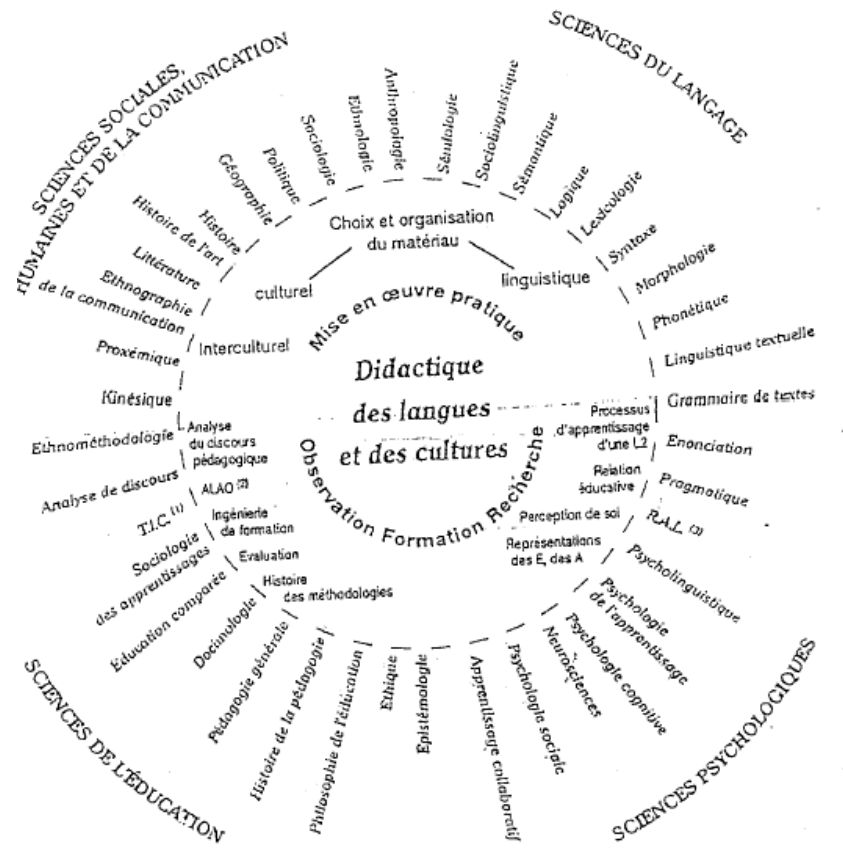

Source : Pothier, 2001, p. 392 
Ces études des "backgrounds " ou ancrages disciplinaires, même si ces derniers ont évolué depuis, permettent d'expliquer en partie les tensions conceptuelles qui règnent dans le champ de la didactique des langues en Suisse (sans doute ailleurs également) et que l’on pourrait catégoriser - avec prudence - comme suit :

- une prédominance des courants psycho(neuro)linguistiques et interactionnistes (dont le plus souvent l'analyse conversationnelle) pour les didacticiens du DaF et de l'EFL, s'appuyant sur les études menées en Applied Linguistics, tandis que du côté FLE, on trouve les courants rattachés aux sciences du langage ainsi qu'aux sciences sociales (micro-sociologie, anthropologie, ethnographie de la communication, anthropologie de la communication, etc.), s’appuyant sur des recherches principalement écrites en français;

- d'un côté, nous aurions affaire à une didactique du "modèle ", dénommée aussi didactique "fonctionnaliste » ou "applicationniste», et de l'autre à une didactique de la "démarche » ou du " processus », désignée dès 2001 par didactique « conceptualiste » (Pothier);

- d'un côté, nous aurions affaire à une didactique aux concepts perçus comme universels et donc transférables imposés d'en haut; du côté FLE, nous aurions affaire à une sociodidactique contextualisée dans l'espace et le temps, émergeant du terrain, aux concepts pas forcément transférables ou qui nécessiteraient en tout cas des aménagements;

- enfin, deux écoles s'opposent nettement dans le traitement de la communication : une conception de langues en contacts, issue de sociolinguistes anglo-américains, et une conception de langues en conflits, issue de sociolinguistes français (Boyer, 1991 et 1997;Gardy et Lafont, 1981), qui sollicitent des postures scientifiques et des conceptions différentes de la communication ainsi que du rapport aux langues.

\section{Effets de la nouvelle doxa : une lutte entre légitimités ou une lutte pour la légitimité?}

On constate, depuis l'implantation des principes de Bologne, l'apparition de directives pour le moins contradictoires venant d'une classe politique suisse souvent en collision, parfois en désaccord, avec une classe économico-financière au pouvoir qui se déclare " néolibérale », et qui encourage simultanément :

- «la promotion de la pluralité linguistique et culturelle» (illustrée par le coûteux financement d’un Centre national de compétences en langues qui a échu à l’Université de Fribourg);

- la généralisation officielle de l’anglais comme langue principale de communication scientifique.

Barbara Cassin, dans son introduction au Dictionnaire des Intraduisibles (2004), réagit à ce phénomène et dit :

C'est le tout anglais, ou plutôt le "tout-à-l'anglais ". Cet anglais officiel de la Communauté européenne et des colloques scientifiques, qui fonctionne certes, mais qui n'est presque plus une langue (les « vrais » Anglais sont ceux qu'on a le plus de mal à comprendre)...

Mais ce "tout-à-l'anglais » (terme auquel nous souscrivons) ne touche pas simplement en Suisse la vie académique et scientifique. Elle touche désormais TOUS les secteurs de la vie publique, politique, économique, médiatique, et fragilise ces nouvelles légitimités durement conquises par ces jeunes didactiques... Quels sont les effets de cette nouvelle doxa sur le champ de la didactique des langues?

\section{Une redistribution de la carte des langues}

Jusqu'ici la Confédération suisse avait délégué la mise en œuvre de l'apprentissage/enseignement de la langue des voisins (ou langues nationales) aux cantons. Depuis les années 2000, cela est devenu une affaire nationale : pour renforcer l'apprentissage des langues nationales, ou ce que nous désignons par "plurilinguisme légitime ", chercheurs et praticiens conçoivent une "didactique intégrée " comprenant à la fois l'anglais et l'enseignement des matières dans une langue étrangère, ce qui est désigné par la notion « d’immersion ».

Parallèlement, l'arrivée au pouvoir d'une droite ultra-libérale de Zurich parachève le processus et met fin à la réciprocité de l'apprentissage de la langue du voisin (français, allemand et italien), en promouvant l'anglais contre les autres langues nationales en situation minoritaire : un véritable raz-de-marée sur toute la Suisse, à tous les niveaux de l'éducation, dans tous les secteurs de la vie économique. 
Et même l'Université bilingue (français-allemand) de Fribourg pratique, de manière encore officieuse, depuis quelques années, un bilinguisme pour le moins curieux : allemand-anglais, et dans une moindre mesure françaisanglais, qui remplacent peu à peu le bilinguisme traditionnel (français-allemand), après avoir abandonné l'italien à son triste sort de langue minoritaire, ici minorée. Le Centre de compétences pour les langues nationales, attribué en 2010 par la Confédération suisse à l'Institut du plurilinguisme de l'Université de Fribourg ${ }^{8}$, semble, malgré ses " missions », renforcer ces tendances.

Ces prises de position officielles, même si les décisions en politiques linguistiques et éducatives relèvent des gouvernements cantonaux, ont des conséquences qui ne se sont pas fait attendre sur le domaine éducatif (dans son sens large). Cette vision harmonieuse et euphorique d'une égalité des chances pour les langues nationales intégrant l'anglais ne résiste pas à l'irrésistible implantation du tout-anglais avec pour conséquences observables :

- disparition progressive du FLE comme première langue nationale enseignée au niveau du primaire dans la majorité des cantons suisses allemands, sauf dans 6 cantons frontaliers, où il est enseigné plus tôt;

- recul évident des langues nationales (allemand, français et italien) dans les cursus académiques - recul annoncé d'ailleurs par Calvet dès 2002 - : à l'Université de Lausanne, à l'EPFL et même à l'EZTH et dans les universités de St-Gall et de Lugano (en Suisse italienne), l'anglais est imposé par les rectorats dans certaines disciplines (sciences dures, sciences de l'environnement, sciences de la matière, économie, gestion et marketing, communication et information, etc.), et les cursus Master mais aussi Bachelor se feront sous peu totalement en anglais, souvent contre l'avis des professeurs (Usunier, 2009);

- disparition entérinée de l'enseignement de l'italien à la fois comme langue nationale, langue seconde et langue étrangère, enseignement de moins en moins offert dans les établissements du secondaire sauf dans les régions frontalières et au niveau universitaire pour de futurs spécialistes de la langue et littérature (par exemple, une didactique de I'ILS [italien langue seconde] que nous avons tenté de promouvoir à Fribourg et en Suisse n'a même pas eu le temps d'éclore);

- constitution d'une nouvelle discipline, officiellement soutenue par la Confédération, le plurilinguisme, dont la grande majorité des acteurs, issus de la linguistique, privilégient l'anglais comme langue scientifique et véhiculent les conceptions de l'Applied Linguistics (ce terme est improprement traduit en français par « linguistique appliquée », voir à ce propos l'introduction de Zarate, Lévy et Kramsch, 2008).

\section{De nouveaux critères de légitimation scientifique}

Nous avons trouvé un grand nombre similitudes entre nos observations et les résultats d'une étude menée par Tania Ogay (Ogay, Zharkova Fattore, Gakuba, Gendre-Borruat, Gremion et Hutter, 2008) sur les recherches en interculturel et leur statut selon que l'étude émane de chercheurs germanophones ou francophones en Suisse. L'analyse minutieuse et comparée des évaluations des projets de recherche retenus par le Fonds national suisse de la recherche scientifique (FNS) a en effet permis à Ogay de distinguer les disciplines et les démarches méthodologiques qui étaient nettement privilégiées.

Nous les reprendrons à notre compte ci-dessous pour le domaine des langues et des cultures :

- prévalence des démarches quantitatives avec analyses descriptives sur les démarches qualitatives et interprétatives propres aux sciences sociales: le mesurable et le chiffrable seraient garants de scientificité (par exemple, l'une de mes doctorantes a posé sa candidature au FNS et a été évaluée très positivement par deux experts francophones, tandis que l'expert germanophone réclamait du quantitatif, percevant la démarche qualitative comme « incomplète »);

- certaines disciplines, et les méthodologies qui leur sont liées, sont considérées comme plus légitimes que d'autres par certains organismes scientifiques, situés à Berne, et évaluateurs des projets de recherche: on constate par exemple, la suprématie de démarches que l'anthropologue Giordano qualifie de «néopositivistes ", comme le psycho-neurocognitivisme, s'appuyant parfois sur de l'imagerie neuronale, qui serait

\footnotetext{
${ }^{8}$ En accordant des soutiens substantiels en millions de francs suisses, en termes de postes, de projets, de conférences, etc., projets pour promouvoir le plurilinguisme de la Suisse.
} 
garante d'une plus grande "scientificité " pour décrire les processus d'apprentissage/d'acquisition des langues;

- suprématie des courants interactionnistes dont l'ethnométhodologie, pas celle du raisonnement sociologique pratique, mais celle de l'analyse conversationnelle de corpus verbaux, le plus souvent décontextualisée, c'està-dire ne prenant pas en compte dans l'analyse des interactions les divers paramètres contextuels, situationnels et statutaires ou autres pouvant jouer un rôle non négligeable dans la co-construction du « sens commun » en langues étrangères ou dans un contexte étranger;

- enfin, la majorité des projets de recherche, dans un pays constitutionnellement plurilingue, sont écrits en anglais; on recrute d'ailleurs de plus en plus de collaborateurs spécialisés dans la rédaction de ces projets, dont le discours scientifique est convenu, avec des thèmes plus "linguistico-politiquement corrects " que d'autres.

Cette nouvelle doxa a pour conséquence de maintenir à distance les sciences sociales, notamment l'anthropologie ou la sociologie, dans le domaine des langues et des cultures, et de considérer les approches qualitatives interprétatives comme suspectes - du moins par certains experts adeptes du quantitatif. Or l'attribution des postes et subventions pour la recherche passe par des commissions fédérales en majorité germanophones... Par ailleurs, ces nouveaux critères de sélection-légitimation contribuent à l'émergence d'une nouvelle catégorie d'acteurs sur la scène académique locale et scientifique internationale, qui calquent leurs attitudes de recherche sur la représentation (souvent fantasmée) d'un "modèle anglo-américain", ce qui a des effets tangibles sur les structures et les individus.

\section{Conclusions}

Nous l'avons vu, promouvoir le respect et la promotion de la diversité peuvent produire des résultats complètement opposés aux finalités, aux enjeux et aux intérêts des institutions et des individus; tenir des discours "politico-linguistiquement corrects" (Calvet, 2002), qui prônent l'égalité des langues et cultures tout en refusant de prendre en compte les rapports de force existant entre langues selon les contextes, les institutions, les champs ou autres, risque d'évacuer toute complexité et même de tuer le plurilinguisme.

Ni le plurilinguisme, ni (et encore moins) le " respect de la diversité linguistique et culturelle " ne peuvent donc s'imposer ou se déclarer de facto simplement parce que quelques politiciens ou chercheurs en mal d'égalité, ou quelques didacticiens en mal de langues, instrumentalisent les langues au service d'une idée: il ne faut pas confondre militantisme et recherche.

Par ailleurs, ces tendances observées dans le champ scientifique en Suisse, à travers le prisme de la didactique des langues et cultures - ou plutôt des didactiques des langues, et les luttes de légitimités qu'elles impliquent entraînent non pas la pluralité des points de vue et des disciplines, comme on se plaît à le proclamer, mais exactement le contraire : soit le formatage scientifique à un type de discours et à des positionnements conceptuels, une "pensée unique " qui s'auto-conforte dans un processus circulant de prophétie auto-réalisante (Wacquant, 2001).

Cette identification des ancrages scientifiques sous-jacents nous invitent donc à la prudence et nous conduisent à décoder les conceptions, les interprétations, les détournements des concepts empruntés aux autres disciplines et de ne pas considérer leur traduction, de manière naïve, comme un fait acquis (Zarate et Liddicoat, 2009); d'un autre côté, cette identification nous oblige à prendre en compte les processus en jeu comme les rapports de force, les (non) légitimités, les reconnaissances, les exclusions, les instrumentalisations. Elle nous invite enfin à postuler la complexité dans nos analyses, ce qui implique de fait des postures plurielles, dans une perspective diachronique et synchronique, attitudes qui peuvent se décliner comme suit :

- le doute; le questionnement;

- l'examen et le réexamen;

- la contextualisation; I'historicisation; 
- l'observation; l'exploration;

- le diagnostic; la comparaison;

- la décentration; la distanciation.

L'ensemble de ces attitudes de recherche promeut l'analyse de l'implicite et davantage encore de celle de "l'impensé ", ou comme nous l'avons déjà écrit du "déjà pensé " (Gohard-Radenkovic, 2009). Si nous voulons concevoir une analyse distanciée, il s'agit de savoir de quel plurilinguisme et de quel pluriculturalisme nous parlons, et à quel niveau, dans quel contexte, dans quelle situation, à quelle époque, et surtout à partir de quel ancrage disciplinaire et dans quelle perspective scientifique ces termes sont apparus...

Enfin, nous l'avons vu, cette imposition du "tout-à-l'anglais » (Cassin, 2004) occulte l'analyse nécessaire des héritages, postures, conceptions et idéologies véhiculés par cette langue et leur impact ou métissages avec d'autres système(s) de pensée.

Dans ce sens, nous rejoignons Cassin (2004) - dont la posture n'est pas sans rappeler la philosophie, la démarche et les objectifs du Précis du plurilinguisme et du pluriculturalisme (Zarate, Lévy et Kramsch, 2008) - quand elle écrit :

L'un des problèmes les plus urgents que pose l'Europe est celui des langues. On peut envisager deux types de solution: choisir une langue dominante dans laquelle se feront désormais les échanges - un anglo-américain mondialisé; ou bien jouer le maintien de la pluralité, en rendant manifestes à chaque fois le sens et l'intérêt des différences, seule manière de faciliter réellement la communication entre les langues et les cultures. Le Vocabulaire européen des philosophies, Dictionnaire des Intraduisibles s'inscrit dans la seconde optique. Mais il regarde vers l'avenir plutôt que vers le passé : il n'est pas lié à une Europe rétrospective et chosifiée - laquelle d'ailleurs? - définie par un cumul d'héritages juxtaposés qui renforcerait les particularismes, mais à une Europe en cours, en activité, energeia plutôt que ergon, qui travaille les écarts, les tensions, les appropriations, les contresens, pour mieux se fabriquer [...]. Une multiplicité n'est pas seulement entre les langues, mais en chaque langue. Une langue, telle que nous l'avons considérée, n'est pas un fait de nature, un objet, mais un effet pris dans l'histoire et la culture, et qui ne cesse de s'inventer - derechef energeia plutôt que ergon.

Nous sommes devant "le risque d'une pensée unique exprimée dans une langue unique » (Gobin, 2011), sorte de " prêt-à-penser » avec ses schémas, ses références et ses modèles pré-formatés et donc reproductibles. Ceci n'est pas sans nous rappeler le roman de science-fiction 1984 de George Orwell, écrivain qui a imaginé un monde totalitaire avec une «novlangue » reposant sur une totale inversion du sens ordinaire comme « la guerre, c'est la paix ", "la liberté, c'est l'esclavage ", "l'ignorance, c'est la force » (Bihr, 2011). Serons-nous un jour amenés à penser comme une évidence que « la pluralité, c'est l'anglais »?

Ces exemples invitent les didactologues des langues et cultures à travailler en amont ces discours implicites et ces dispositifs officiels "qui promeuvent le plurilinguisme et le pluriculturalisme » et instrumentalisent les langues à des fins idéologiques, politiques et économiques, au service de la nouvelle doxa du tout-anglais, légitimée par les acteurs même de l'institution scientifique. Nous sommes bien au cœur non pas d'une guerre des langues mais d'une guerre des légitimités.

\section{Références bibliographiques}

Androulakis, G. (2003). La Grèce, terre d'accueil ou l'émergence d'un nouvel espace interculturel. Dans A. GohardRadenkovic, D. Mujawamarija et S. Perez (dir.), Intégration des «minorités» et nouveaux espaces interculturels. Berne : Peter Lang.

Bausch, K., Christ, H., Königs, F. et Krumm, H.-J. . (dir.) (1996). Erforschung des Lehrens und Lernens fremder Sprachen. Zwischenbilanz und Perspektiven. Tübingen : Gunther Narr Verlag.

Bihr, A. (2011). L'idéologie néo-libérale. Revue de sémio-linguistique des textes et discours, SEMEN, 30, 1-16. (Les langages de l'idéologie. Études pluridisciplinaires)

Bourdieu, P. (2002). Introduction : les conditions sociales de la circulation internationale des idées, Actes de la recherche en science sociales (ARSS), 145, 3-5. 
Bourdieu, P. (1980). Le sens pratique. Paris : Éditions de Minuit.

Boyer, H. (1991). Langues en conflit. Paris : L'Harmattan.

Boyer, H. (dir.) (1997). Plurilinguisme : " contact » ou « conflit » de langues? Paris : L'Harmattan.

Calvet, L.-J. (2002). Le marché aux langues, Paris : Plon.

Cassin, B. (dir.) (2004). Le vocabulaire de la philosophie. Le dictionnaire des intraduisibles. Le Robert/Seuil.

Clark, A. (2008). Background Project. Report on Interviews carried out with Teaching staffs in 2007 (Enquête non publiée). Fribourg : CERLE / Université de Fribourg.

Conseil de l'Europe (2008). Guide pour l'élaboration des politiques linguistiques éducatives en Europe. De la diversité linguistique à l'éducation plurilingue / Guide of the Development of Language Education Policies in Europe. From linguistic Diversity to plurilingual Education. Strasbourg : Conseil de l’Europe.

Edmondson, W. et House, J. (2000). Einführung in der Sprachlehrforschung (2 éd.). Tübingen/Bâle : A. Francke.

Galisson, R. (2002). Préambule : est-il fou? Est-il sage? Comment peut-on être didactologue? ELA. Études de linguistique appliquée, 127, 261-271. http://www.cairn.info/revue-ela-2002-3-page-261.htm

Galisson, R. et Porcher, L. (coord.) (1985). Didactologies et idéologies. ELA. Études de linguistique appliquée, 60.

Gardy, P. et Lafont, R. (1981). La diglossie comme conflit : l'exemple occitan. Langages, 61, 75-91.

Giordano C. (2008). Postface. L'insoutenable innocence de l'interculturel. Dans A. Gohard-Radenkovic et A. Akkari (dir.), La coopération internationale: entre utopies du changement et accommodements interculturels (p. 161-170). Paris : L'Harmattan.

Gobin, C. (2011). Les principales caractéristiques du discours politique contemporain. SEMEN, Revue de sémiolinguistique des textes et discours, 30,1-16. (Les Langages de l'idéologie. Études pluridisciplinaires)

Gohard-Radenkovic, A. (dir.) (2005). Plurilinguisme, interculturalité didactique des langues étrangères en contexte bilingue/Mehrsprachigkeit, Interkulturalität, und Fremdsprachendidatik im zweisprachigen Kontext $\left(2^{\mathrm{e}}\right.$ éd., 2008). Berne : Peter Lang.

Gohard-Radenkovic, A. (2006). Interrogations sur la dimension interculturelle dans le Portfolio européen des langues et autres productions du Conseil de l'Europe. Synergies Europe, 1, 82-94. (La richesse de la diversité : recherches et réflexions dans l'Europe des langues et des cultures)

Gohard-Radenkovic, A. (2007). Didactique des langues étrangères/Sprachlehrforschung. Des théories aux pratiques : un champ en question(s). Dans J-L. Patry et C. Giordano (dir.), Theorie und Praxis: Brüche und Brücken, Freiburger Sozialanthropologische Studien / Fribourg Studies in Socia Anthropology. Münster : LiT Verlag.

Gohard-Radenkovic, A. (2009). Peut-on former à la différence? Peut-on « penser la différence » dans la mobilité? Les Cahiers de I'APLIUT - Pédagogie et recherche, 28(2), 11-23. http://apliut.revues.org/1041

Gohard-Radenkovic, A. (2010). Politiques de gestion de la pluralité linguistique : leurs effets sur les logiques des institutions et les logiques des individus. Dans F. Ruegg et A. Boscoboinik (dir.), From Palermo to Penang. A Journey into Political Anthropology/De Palerme à Penang. Un itinéraire en anthropologie politique. Hommage à Christian Girdano (p. 119-135). Berlin : LiT Verlag.

Lenz, P. et Berthele, R. (2010). Prise en compte des compétences plurilingue et interculturelle dans l'évaluation, Strasbourg : Conseil de l'Europe.

Maurer, B. (2011). Enseignement des langues et construction européenne. Le plurilinguisme, nouvelle idéologie dominante. Paris : Editions des Archives contemporaines.

Ogay, T., Zharkova Fattore, Y., Gakuba Th., Gendre-Borruat S., Gremion M., et Hutter V. (2008). La recherche empirique en éducation interculturelle en Suisse : comparaison entre la Suisse francophone et la Suisse germanophone. Revista Espanola de Educacion Comparada, 14, 177-197. 
Porcher, L. (1987). Champs de signes. Etats de la diffusion du Français langue étrangère. Paris : Crédif /Didier.

Pothier, M. (2001). Formation à la recherche et recherche de la formation en didactique, ELA. Études de linguistique appliquée, 3(123-124), 385-392. En ligne à : http://www.cairn.info/revue-ela-2001-3-page-385.htm

Tabouret-Keller, A. (2011). Bilinguisme en procès (1840-1940). Limoges (France) : Editions Lambert-Lucas.

Usunier, J.-P. (2009). Un pluriliguisme pragmatique face au mythe de l'anglais lingua franca de l'enseignement supérieur. Conférence présenté au colloque de l'Académie suisse des sciences humaines et sociales, Les enjeux du plurilinguisme pour la construction des savoirs, Berne, Suisse.

Wacquant, L. (2001). Sur l'Amérique comme prophétie auto-réalisante. ARSS, Actes de la recherche en sciences sociales, $139,86-87$.

Widmer, J., Coray, R., Acklin Muji, D. et Godel, É. (2004). Die Schweizer Sprachenvielfalt im öffentlichen Diskurs/La diversité des langues en Suisse dans le débat public ( 2 éd.). Berne : Peter Lang.

Zarate, G. et Liddicoat, A. (coord.). (2009). La circulation internationale des idées en didactique des langues. Paris : FIPF / CLÉ international. (Coll. Recherches et Applications / Le français dans le monde).

Zarate, G., Lévy D. et Kramsch, C. (2008). Introduction. Dans G. Zarate, D. Lévy et C. Kramsch (dir.), Précis du plurilinguisme et du pluriculturalisme (p. 5-11). Paris : Éditions des Archives contemporaines. 